\title{
L'exploitation du lignite et la réhabilitation des paysages dans la région minière d'Olténie en Roumanie
}

Extraction of lignite and remediation of landscapes in Oltenia mining region in Romania

Braunkohlenbergbau und Rekultivierung der Bergbaulandschaften in dem Revier Oltenien in Rumänien

\section{Corina Calugaru}

\section{(2) OpenEdition}

Journals

Édition électronique

URL : http://journals.openedition.org/rge/1444

DOI : $10.4000 /$ rge. 1444

ISSN : 2108-6478

Éditeur

Association des géographes de l'Est

Édition imprimée

Date de publication : 1 juin 2006

ISSN : 0035-3213

\section{Référence électronique}

Corina Calugaru, «L'exploitation du lignite et la réhabilitation des paysages dans la région minière d'Olténie en Roumanie », Revue Géographique de l'Est [En ligne], vol. 46 / 3-4 | 2006, mis en ligne le 18 décembre 2009, consulté le 08 septembre 2020. URL : http://journals.openedition.org/rge/1444 ; DOI : https://doi.org/10.4000/rge.1444

Ce document a été généré automatiquement le 8 septembre 2020.

Tous droits réservés 


\title{
L'exploitation du lignite et la réhabilitation des paysages dans la région minière d'olténie en Roumanie
}

\author{
Extraction of lignite and remediation of landscapes in Oltenia mining region in \\ Romania \\ Braunkohlenbergbau und Rekultivierung der Bergbaulandschaften in dem \\ Revier Oltenien in Rumänien
}

Corina Calugaru

\section{Introduction}

1 Bien que l'industrie minière ait une tradition ancienne en Roumanie, les plus grandes transformations paysagères ont été apportées par la mise en place des exploitations de lignite. Située entre les vallées de l'olt à l'est et du Danube à l'ouest, dans une région collinaire et de plateau, la région minière d'olténie est la région la plus profondément marquée par les exploitations de lignite à ciel ouvert. Le lignite représente une ressource énergétique relativement abondante dans le pays. Les réserves totales, estimées par le Ministère de l'Industrie et du Commerce en 2000, sont de 3,0 Gt, 90 \% $(2,5 \mathrm{Gt})$ de ces réserves se situant dans les limites de cette région. Ces ressources sont liées à l'évolution géologique du bassin sédimentaire Gétique, un bassin situé au pied des Carpates Méridionales, à la fin du Néogène et au début du Quaternaire.

Le développement de cette industrie s'est faite d'une façon plus récente mais, néanmoins, aussi brutale que dans d'autres régions minières d'Europe. Durant moins d'un demi-siècle d'exploitation, le lignite a créé non seulement un type de paysage avec un fonctionnement et des caractéristiques particulières mais aussi une économie locale et une société totalement dépendante des carreaux de la mine. Ce type de paysage 
caractérisé par une dynamique particulière, accélérée, entièrement artificielle, imposée par la mine et que l'on retrouve à travers toute l'Europe, justifie l'appartenance de cette région à la grande famille des "pays noirs" (Baudelle, 1994). On retrouve non seulement des paysages qui se ressemblent mais aussi la même culture, la culture de la solidarité sociale, des syndicats, de la prospérité mais aussi des conditions de travail insalubres et dangereuses, une culture accompagnée de perceptions et de représentations propres à la communauté minière.

3 Même si l'exploitation du lignite a servi de support au développement économique, social et culturel de cette région, elle est aussi responsable des profondes transformations paysagères et des graves problèmes environnementaux.

Il s'agit de comprendre comment est né ce paysage minier dans une région totalement agricole, quelles sont les mutations qui se sont produites et comment celles-ci ont structuré un nouveau paysage. D'autre part, on s'interrogera sur les actions menées pour reconstruire un paysage totalement détruit par les exploitations minières et en particulier si on peut utiliser l'expérience d'autres pays européens pour la reconstruction du paysage minier en Roumanie, où l'intérêt pour l'environnement a été quasi inexistant sous l'ancien régime communiste.

\section{Le contexte du développement des exploitations}

5 Si l'on regarde au-delà des symboles, des perceptions ou des représentations, on s'aperçoit que cette région minière a aussi ses particularités, des particularités imposées par le cadre humain, économique et politique. Chaque société modèle son espace et imprime une certaine dynamique au paysage, dynamique qui se traduit par des formes et des structures spatiales qui servent de cadre de vie à cette société. Dans l'organisation spatiale du paysage et dans son fonctionnement on retrouve des idéologies, des lois et des politiques qui caractérisent chaque société à un moment donné.

6 Le développement des exploitations de lignite dans la région d'Olténie s'est faite après les années cinquante, précisément dans une période où, dans la plupart des pays européens, l'extraction du charbon n'était plus considérée comme rentable et la fermeture des mines commençait. Le territoire des anciens bassins miniers est ainsi devenu l'un des plus grands chantiers de reconstruction, réhabilitation et de reconversion industrielle. De nouvelles questions se sont posées, relatives à la sécurisation et à la dépollution des anciens sites, à la reconstruction des paysages et surtout à la reconversion économique et sociale des territoires. Beaucoup de pays ont mis en place des politiques et des programmes destinés à reconstruire et à reconvertir des régions minières en déclin. Parmi les régions minières les plus représentatives, on peut citer la Ruhr, le Nord-Pas-de-Calais, la Silésie, la Sarre, la Lorraine, etc. Même si ces programmes auraient pu servir d'exemple pour une meilleure prise en compte des problèmes environnementaux dans le processus d'exploitation, la Roumanie n'a pas su, ou n'a pas pu, vu le régime politique existant, profiter de ces expériences. Ainsi, une fois les premières exploitations fermées, la région étudiée a plongé, après 1997, dans un contexte socio-économique régressif qui a bouleversé les conditions du développement et qui rendent nécessaires de grands efforts de reconstruction et de reconversion économique et sociale. 
7 Sur le plan temporel, l'activité d'exploitation du lignite est de relativement courte durée, par rapport à d'autres régions d'Europe; les premières exploitations ont commencé en 1956, mais de manière très accélérée, ce qui offre une synthèse des agressions qu'une industrie peut apporter à son environnement sur un horizon de temps réduit. Avant la deuxième guerre mondiale, la Roumanie possédait une image célèbre pour la beauté et la richesse de son milieu naturel (Lhomel, 1984). On passe d'une période caractérisée par le souci de protection d'une flore et d'une faune, aux caractéristiques parfois exceptionnelles, marquée par la création en 1920 de la «société de tourisme pour la protection de la nature", à une période marquée, après la deuxième guerre mondiale, par la surexploitation des ressources naturelles.

8 La Roumanie est un pays qui a subi deux bouleversements politiques majeurs, la nationalisation de 1948 et la révolution de 1989, bouleversements qui ont profondément marqué le paysage. C'est avec la nouvelle politique des années cinquante, et après les pertes subies pendant la deuxième guerre mondiale, que le lignite devient un pilier essentiel pour le développement économique, en assurant une certaine indépendance énergétique du pays. Après les événements de décembre 1989, l'ouverture du pays et la volonté de faire partie des structures européennes et mondiales ont déterminé des changements radicaux, non seulement sur le plan politique mais aussi sur le plan économique, social et environnemental. La montée des préoccupations environnementales au niveau mondial, qui ont abouti au Protocole de Kyoto en 1997, conduit à s'interroger sur la place du charbon dans le bilan énergétique du pays, la Roumanie faisant partie des pays signataires du Protocole de Kyoto. Pendant cinquante années, la mise en valeur et l'exploitation des ressources naturelles a répondu aux seuls impératifs de nature économique, mais dans le nouveau cadre et dans la perspective d'adhésion de la Roumanie à l'Union Européenne, le développement durable s'impose de plus en plus comme cadre normatif en ce qui concerne l'exploitation des ressources naturelles, surtout le charbon. Avec la reconstruction du paysage minier, la Roumanie s'inscrit dans la politique paysagère européenne qui demande, aux niveaux local, régional, national et international, des politiques et des mesures destinées à protéger, à gérer et à aménager les paysages d'Europe afin d'améliorer leur qualité.

9 Ces dernières décennies, même si son image s'est détériorée de plus en plus, à cause de la multitude des problèmes environnementaux qu'il engendre, le lignite continue à occuper une place importante sur le marché énergétique national. 
Figure 1 : La structure de la production d'électricité en Roumanie en 2005

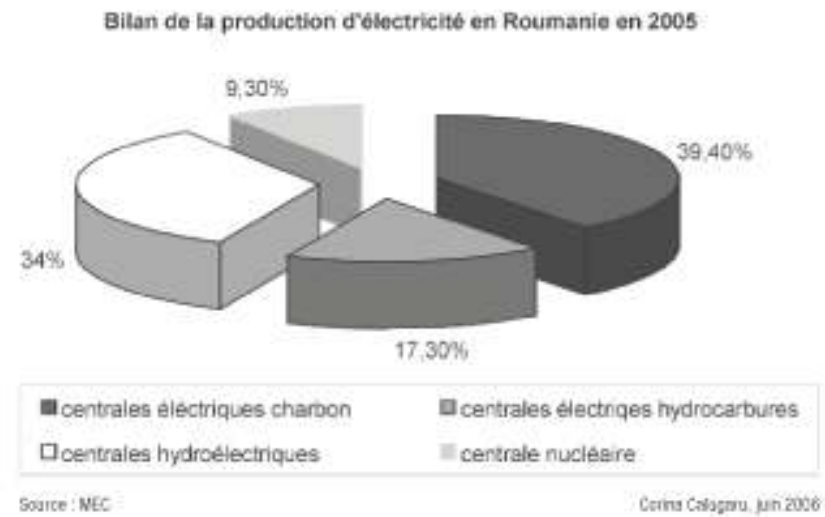

Comme on peut l'observer dans la figure 1, le charbon est, et va continuer à rester, la principale source pour la production d'électricité en Roumanie. Dans la stratégie énergétique du pays pour la période 2002-2015 il est prévu que ce type d'énergie constitue, ensemble avec l'énergie nucléaire, la principale option pour assurer une certaine indépendance énergétique, vu l'augmentation du prix des hydrocarbures et l'insuffisance des ressources nationales de gaz qui rendent le pays dépendant des importations. Même si la production de charbon a baissé après 1989, la proportion du lignite dans la production totale reste constante (Tableau 1, fig. 2). Conformément à la stratégie énergétique du pays, on prévoit une augmentation de la production de lignite pour les années à venir, production qui va se stabiliser à 35 millions de tonnes par an.

Tableau 1 : Évolution de la production de lignite après 1989 (millions de tonnes)

\begin{tabular}{|l|c|c|c|c|c|c|c|c|c|c|c|c|}
\hline Année 1989 & 1993 & 1994 & 1995 & 1996 & 1997 & 1998 & 1999 & 2000 & 2001 & 2002 & 2003 \\
\hline $\begin{array}{l}\text { Charbon } 61,5 \\
\text { total }\end{array}$ & 42,4 & 43,2 & 43,9 & 44,7 & 36,2 & 28,1 & 24,5 & 30,9 & 34,7 & 31,9 & 34,4 \\
\hline Lignite & 53,3 & 36,1 & 36,3 & 37,0 & 37,2 & 30,1 & 23,4 & 20,4 & 26,5 & 30,4 & 27,7 & 30,8 \\
\hline
\end{tabular}

Source: : L'Annuaire statistique de la Roumanie. 
Figure 2 : L'évolution de la production de lignite entre 1989 et 2003

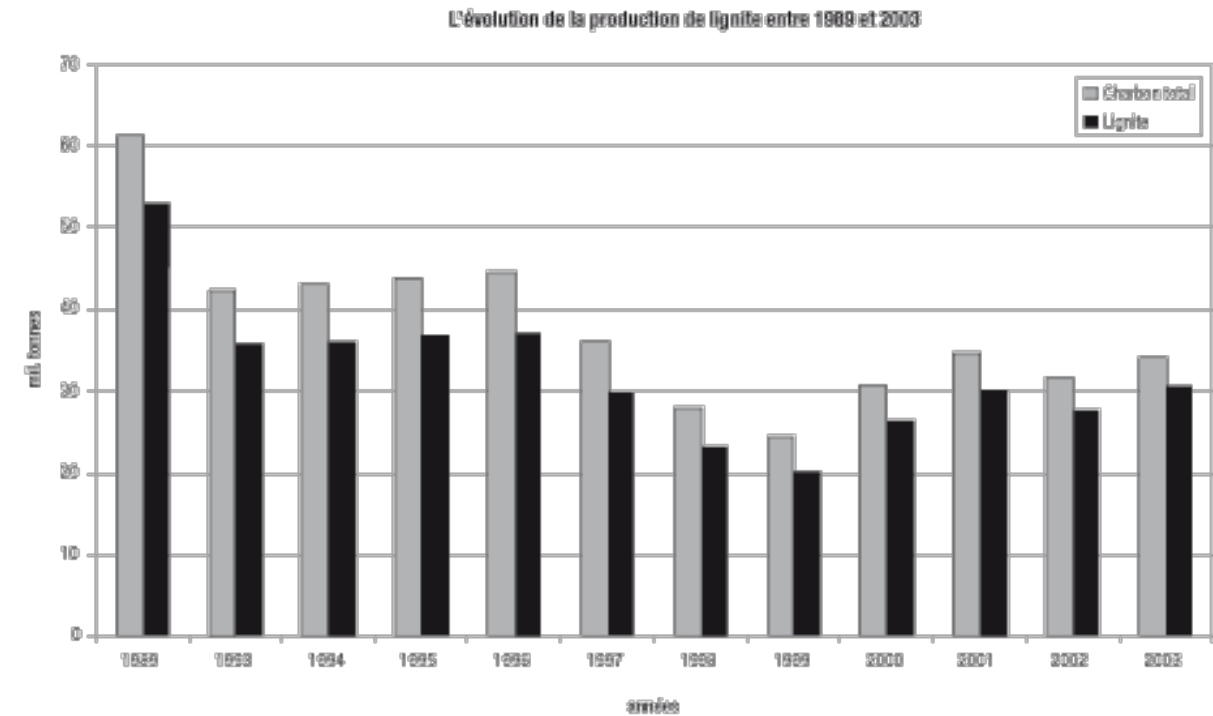

11 Le maintien des exploitations de lignite dans cette région n'a pas seulement des raisons économiques. Il est aussi motivé par la stratégie nationale d'approvisionnement énergétique et des raisons sociales, notamment la stabilité de l'emploi dans une région défavorisée, où la mine reste le principal domaine d'activité; d'autant plus que, le lignite de la région minière d'olténie est considéré, par ses caractéristiques géologiques, permettant l'exploitation à ciel ouvert, comme le bassin charbonnier le plus rentable du pays, dans la mesure où l'activité a cessé d'être subventionnée depuis 1997.

\section{La substitution d'un paysage à un autre}

Le paysage minier a pris place dans une région rurale et a entraîné une dynamique paysagère, économique et démographique accélérée. Dans un intervalle relativement réduit de temps on passe d'un paysage agricole à un paysage ou les structures minières viennent s'insérer et imposer brutalement leur présence.

\section{A. Un vieux paysage agraire}

La région d'Olténie d'avant l'industrialisation, nous offrait un paysage complètement différent de celui d'aujourd'hui. Pendant des siècles, le système de production agricole et les petits villages ont été les seuls qui ont structuré le paysage dans cette région. L'utilisation des terrains s'est constamment modifiée en fonction des nécessités de la population, mais surtout en fonction de la nature des relations sociales et du niveau de développement économique.

$\mathrm{Du} \mathrm{XV}^{\mathrm{e}}$ au $\mathrm{XVII}{ }^{\mathrm{e}}$ siècles cette région était considérée comme l'une des principales régions de production agricole des Pays Roumains, ce qui a permis la maturation des villages, la différenciation des villes et la cristallisation des premières formations étatiques et religieuses féodales.

L'agriculture a été l'activité prédominante pendant des siècles. De vieux documents cartographiques ${ }^{1}$ montrent que la plus grande partie de la surface était occupée par des 
forêts (pratiquement les deux tiers), des pâturages et des prairies, les terrains cultivés occupant des surfaces réduites, ce qui atteste le caractère agro pastoral des activités. Les surfaces cultivées en céréales occupaient surtout les dépressions.

À la fin du XVIII e siècle, le droit de libre usage accordé aux paysans, au début du même siècle, pour l'élevage du bétail sur des terrains non cultivés, commence à être restreint, et les terrains cultivés se développent au détriment des forêts. Cette situation atteste un changement de propriété, et le passage des relations de type féodal à des relations de type capitaliste. Les activités pastorales, caractéristiques aux structures féodales sont remplacées par des activités céréalières, représentatives pour les structures capitalistes, qui changent la physionomie et le fonctionnement du paysage. On trouvait des cultures céréalières dans les dépressions et les vallées, les versants étant le domaine des forêts, des pâturages et des prairies. Pendant le XIX ${ }^{e}$ siècle, sur les versants exposés au sud, est ou ouest, des vignobles et des vergers ont remplacé les forêts, d'autant plus que la région bénéficie d'un climat abrité et la vigne sauvage se développe spontanément.

Depuis le début du $\mathrm{XX}^{\mathrm{e}}$ siècle, le morcellement des terrains et la petite propriété paysanne deviennent le trait dominant de la structure agraire. La majorité des exploitations a une surface inférieure à un hectare, chaque famille possède un attelage, ce qui lui permet de travailler ses terres, pour son propre compte. Avant la deuxième guerre mondiale la Roumanie toute entière restait encore un pays peu industrialisé, les trois quarts de la population et $58 \%$ du revenu national étant assurés par l'agriculture. Cette situation était due à l'évolution historique de la Roumanie, quifut un protectorat turc pendant des siècles, mais aussi à des accords économiques comme le traité d'Andrinople de 1829, qui favorisait l'exportation de céréales, surtout le blé.

Les plus grandes transformations du paysage agraire se produisent après la deuxième guerre mondiale, avec le changement du système politique et la naissance du « Partidul Muncitoresc Român » (PMR), Parti Ouvrier Roumain, lors du Congrès de février 1948. Les nouveaux acteurs sur la scène politique ont pris la décision de la collectivisation, adoptée par l'Assemblée Plénière du parti en mars 1949. Le processus a démarré en 1950 et a duré treize années, jusqu'en 1962. Diverses mesures visant à « convaincre » les paysans ont été prises. La collectivisation forcée a abouti à la mise en place de deux types de structures institutionnelles, les CAP, les coopératives agricoles de production, et les IAS, les entreprises agricoles d'état. Elles ont modelé un nouveau paysage agraire qui avait comme principale caractéristique le remembrement, ce qui a déterminé des transformations de la couverture végétale et une augmentation du ruissellement. Une fois de plus, la logique économique l'emporte sur la logique environnementale. Seuls les terrains situés dans les montagnes ou dans des régions collinaires, avec un relief fragmenté, difficile à mécaniser, sont restées en propriété privée et ont fait la richesse de ces régions.

19 C'est sur ce fond de conflits et de vives tensions, au sein d'une société agricole, que se sont opérées les profondes mutations sociales et économiques de ces villages consécutives à la mise en place des exploitations de lignite.

\section{B. Une région aux limites floues}

La région minière d'olténie n'a pas de limites naturelles ou administratives, c'est une région aux limites floues, l'élément qui impose la dynamique étant le charbon. Du point 
de vue administratif elle est partagée entre trois départements : Mehedinti, à l'ouest, Gorj au centre, qui détient la majorité des exploitations, et Vâlcea à l'est.

Figure 3 : Le découpage administratif de la région minière d'Olténie

LA REGION MINIERE OLTENIE : LES COMMUNES MINIERES

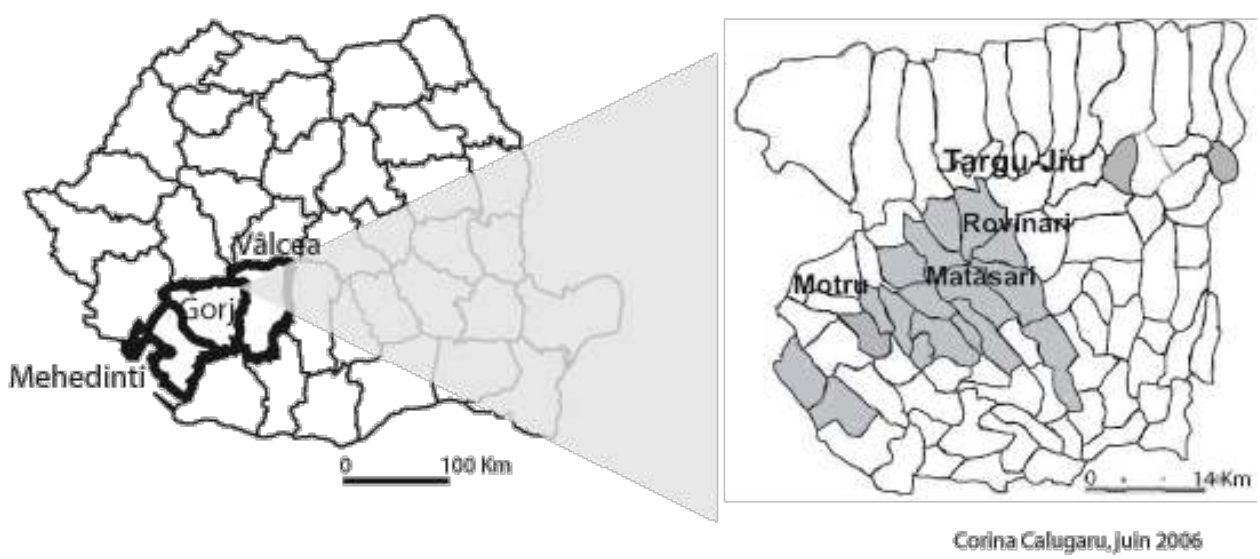

Dans l'image de gauche est représenté le découpage administratif du pays avec les trois départements où se situent les exploitations minières de la région d'Olténie. Dans l'image de droite sont représentées, au milieu, les communes minières du département de Gorj et les deux communes minières du département Mehedinti.

Dans la figure 3 sont représentées uniquement les communes minières des départements de Gorj et de Mehedinti, celles du département de Gorj, situées au centre de l'image, étant représentées par les plus grandes exploitations à ciel ouvert du pays. Souvent, les carrières et les terrils dépassent les limites administratives des communes et on peut les trouver à cheval sur deux ou plusieurs communes, ce qui rend difficile le processus de reconstruction après la mine.

\section{Un nouveau paysage : des exploitations à ciel ouvert}

La découverte et la mise en exploitation des ressources de lignite ont apporté des transformations radicales au paysage. Les entreprises minières se sont implantées dans ces territoires de tradition agricole et ont commencé à modeler un nouveau paysage. La dynamique spatiale du paysage minier a nécessité le déplacement et la destruction des villages, le détournement des cours d'eau, la modification de la topographie, etc. En quelques années, l'activité dominante n'est plus l'agriculture mais l'extraction et la valorisation du lignite. Les infrastructures minières ont commencé à se substituer progressivement au paysage agraire existant et la configuration du gisement de lignite a conditionné l'extension spatiale du paysage minier (figure 4). 
Figure 4 : le cadre naturel du bassin minier : le piedmont des Carpates méridionales (d'après $\mathrm{E}$. de Martonne, 1931, modifié)

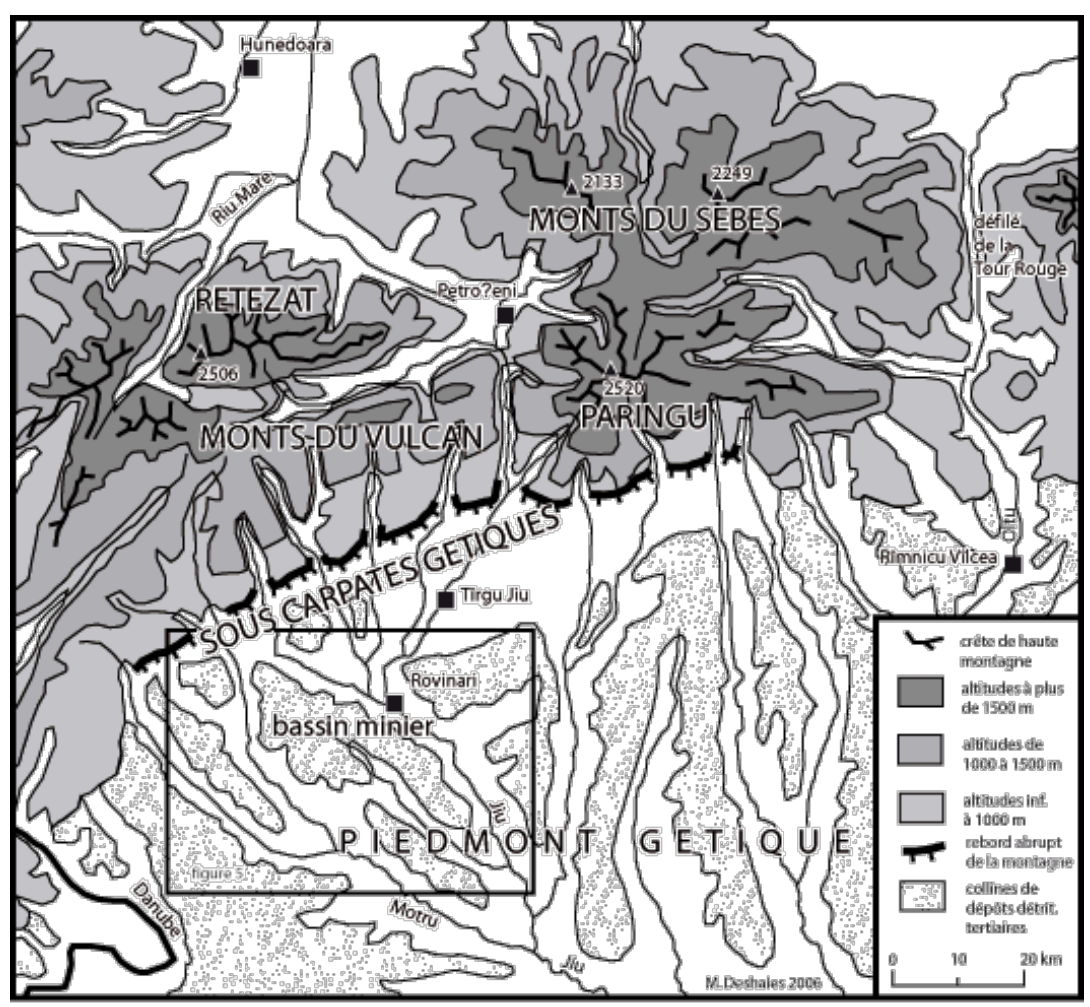

Le cadre naturel dans lequel se sont implantées les exploitations minières correspond à deux types d'unités : au nord les Sous Carpates Gétiques (200-600 m), avec une structure plissée en anticlinal et synclinal s'étirant d'ouest en est. Au sud, se trouve le piedmont Gétique (200-400 m) qui en occupe la plus grande partie, avec une structure monoclinale et une morphologie représentée par des collines orientées du nord-ouest au sud-est, restes d'une ancienne surface de piémont.

L'affaissement progressif, au pied des Carpates, d'un fossé d'effondrement, appelé la Dépression Gétique, depuis le Sénonien et puis la sédimentation jusqu'au Pléistocène, a permis le dépôt d'une succession de 18 couches de lignite qui alternent avec d'autre dépôts sédimentaires (argiles, marnes, sables, etc.) constituant d'importants réservoirs hydrogéologiques. Les couches de lignite les plus anciennes sont enfouies jusqu'à plus de $350 \mathrm{~m}$ de profondeur et les plus récentes sont presque à l'affleurement, dans la vallée du Jiu, et ne sont recouvertes que par quelques mètres de graviers. L'inclinaison des couches est faible, elle ne dépasse pas $5-10^{\circ}$, et l'épaisseur pour chaque couche de lignite varie entre 1,0 et $8,0 \mathrm{~m}$. Le complexe carbonifère entier a une épaisseur de 10-30 m dans l'espace des vallées et jusqu'à $120 \mathrm{~m}$ dans l'espace collinaire. Par ces caractéristiques et pour des raisons de rentabilité économique, le lignite du bassin minier d'Olténie est exploité en grande partie $(80 \%)$ à ciel ouvert et est utilisé pour l'alimentation des centrales thermiques de grande capacité, Turceni et Rovinari, construites à proximité.

Dans le processus d'occupation de l'espace, l'entreprise minière dirigée par l'Etat, et qui à son tour exerçait le pouvoir sur les exploitations minières, dans un système d'emboîtement d'échelles, prenait toutes les décisions administratives, économiques, financières, juridiques et sociales.

Le territoire a été partagé entre plusieurs exploitations minières, de manière à avoir une réserve foncière suffisante, leur permettant l'exploitation et la mise en place des 
installations techniques nécessaires. Ces exploitations sont réparties sur cinq bassins miniers qui se déroulent plus ou moins en continuité, de l'est à l'ouest, sur une distance de $103 \mathrm{~km}$. A l'extrémité est, on trouve dans un relief collinaire, le bassin de Berbesti qui se situe dans le département de Vâlcea. Au centre, se situent les trois bassins du département de Gorj (figure 4) : le bassin de Rovinari, dans la partie sud de la dépression Târgu-Jiu Câmpu Mare et le long du couloir de la rivière Jiu ; le bassin de Jilt qui s'étend en continuité vers l'ouest, occupant la partie est des collines du Jilt, avec un relief très fragmenté et, toujours en continuité vers l'ouest, le bassin de Motru, occupant la partie ouest des collines du Jilt et le couloir de la rivière Motru. Enfin, à l'extrémité ouest de la région minière, on trouve le bassin de Mehedinti, avec des exploitations d'extension moins importante que celles des autres bassins miniers.

Pour la constitution de la réserve foncière on distingue deux périodes qui, se différencient par leur législation, la période avant 1989 et la période après 1989 (Calugaru, 2004). La situation détaillée pour chaque période et pour chacun des cinq bassins miniers est présentée dans le tableau 2.

Tableau 2 : Les surfaces occupées par l'industrie minière en Olténie

\begin{tabular}{|c|c|c|c|c|c|c|c|c|c|c|c|}
\hline \multirow{3}{*}{$\begin{array}{l}\text { Le bassin } \\
\text { minier }\end{array}$} & \multicolumn{11}{|c|}{ Surface occupée (ha) } \\
\hline & \multirow{2}{*}{$\begin{array}{c}\text { Total } \\
\text { ha }\end{array}$} & \multicolumn{4}{|c|}{ Utilisation initiale } & \multicolumn{3}{|c|}{ 1952-1990 } & \multicolumn{3}{|c|}{$1991-2000$} \\
\hline & & Agricole & $\%$ & Sylvicole & $\%$ & Total & Agricole & Sylvicole & Total & Agricole & Sylvicole \\
\hline Rovinari & 8658 & 7154 & 82,7 & 1504 & 17,3 & 7509 & 6335 & 1174 & 1149 & 815 & 334 \\
\hline Jilt & 2164 & 1730 & 79,9 & 434 & 20,1 & 1796 & 1433 & 363 & 368 & 297 & 71 \\
\hline Motru & 3683 & 2578 & 69,3 & 1105 & 30,2 & 3436 & 2444 & 992 & 247 & 128 & 119 \\
\hline
\end{tabular}

Source : CNLo Oltênie, 2000.

Pour la première période, qui s'est déroulé entre 1950 et 1989, l'Etat, par l'intermédiaire du Conseil d'Etat et du Conseil des Ministres, était le principal acteur législatif. Ainsi, les concessions étaient accordées aux Entreprises minières par des Décrets et Arrêtés. Seuls les terrains qui se trouvaient dans la propriété de la CAP faisaient l'objet d'une compensation. La superficie concédée par l'État dépendait de l'estimation du volume de lignite exploitable. Au total, ce sont 15041 ha qui ont été occupés parmi lesquels 11577 correspondaient à des terrains agricoles et 3464 ha représentaient des forêts.

La deuxième période, qui a débuté après la chute du communisme en décembre 1989, a été marquée par de profondes mutations politiques, économiques et juridiques. "L'âge d'or » de la propriété socialiste, qui caractérisait la période précédente, était désormais révolue et une nouvelle législation en ce qui concerne la forme de propriété sur les terrains a été adoptée. Les terrains agricoles, propriété des CAP, deviennent de nouveau propriété des paysans, et les Compagnes minières sont obligées maintenant de traiter avec eux, ou, dans le cas des forêts, avec ROMSILVA (Régie Nationale des Forêts). Mais, en dépit du changement de propriété, l'emprise minière sur des territoires ruraux se poursuit, des maisons, des terrains agricoles des forêts laissent leur place aux carrières et aux terrils. Durant cette période, ce sont 2164 ha ( 1525 ha de terrains agricoles et 639 ha de forêts) qui sont occupés à la suite de réquisitions ordonnées par le ministère 
en charge qui conduit les négociations directement avec plus de 2500 particuliers et 7 unités de la Régie Nationale des Forêts.

Les conditions topographiques dans lesquelles se sont implantées les exploitations sont très variées : on les trouve dans le lit majeur des rivières, sur des versants, ou sur des collines, comme on peut l'observer dans la figure 3.

L'extraction du lignite a commencé en 1955 avec l'ouverture de trois carrières dans le bassin de Rovinari, au bord de la rivière Jiu, là où les couches de charbon étaient situées près de la surface. Les années qui suivent, les excavations commencent à grignoter l'espace collinaire situé à l'ouest du Jiu. De gigantesques excavations se sont développées aux dépens des terrains fertiles, des forêts, ou même des villages, qui ont été détruits pour permettre la progression des exploitations. Au début, les exploitations à ciel ouvert, ont été équipées avec des technologies simples, représentées par des excavatrices avec des pelles à godets et des camions utilisés pour le transport du charbon et des stériles. A partir des années 1960, des technologies d'extraction continue commencent à être employées dans les grandes carrières, avec des excavatrices gigantesques à pelles rotatives, importées d'Allemagne, et des ponts transporteurs qui permettent de remuer des volumes considérables de matériaux, les technologies classiques continuant à être utilisées dans des carrières plus petites.

31 Dans la région d'Olténie il existe aujourd'hui 12 mines et 19 exploitations à ciel ouvert, la plupart d'entre elles (9) se trouvent dans le bassin de Rovinari, et sont situées dans le lit majeur du Jiu, engendrant des transformations radicales du paysage.

32 La répartition géographique des anciennes surfaces minières tout au long de la rivière Jiu, reflète le stade de la progression du front des exploitations; les plus grandes exploitations se répartissent essentiellement le long d'un axe représenté par la vallée du Jiu et de l'ouest à l'est entre la vallée de la rivière Motru et la vallée de la rivière Jiu. Les anciennes carrières et terrils font aujourd'hui l'objet d'une reconstruction plus intense. Aucune commune située à l'intérieur du bassin minier n'a été ou ne sera épargnée par l'avancement des fronts d'exploitation.

Si regardée de l'extérieur la région d'olténie a un aspect homogène, au regard de sa spécificité économique dominée par l'activité minière, à l'intérieur, le mélange entre les structures minières et les structures anciennes révèle une organisation paysagère morcelée, hétérogène. L'emprise spatiale de l'industrie minière (fig. 5) et des activités connexes a rompu l'équilibre qui existait entre l'activité agricole et son milieu et a déclenché une régression des potentialités écologiques. 
Figure 5 : Le développement des exploitations minières

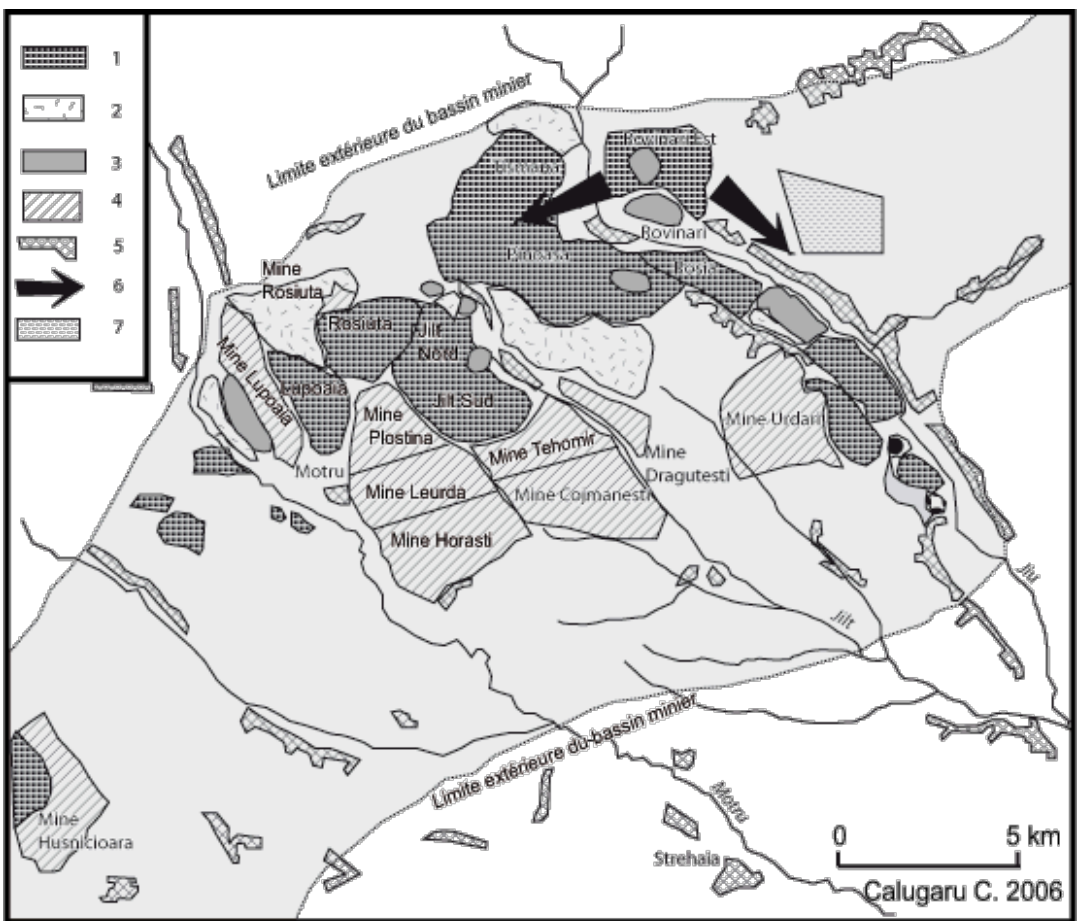

1. carrierres ; 2 . terrils en vole de constitution; 3. terrils amenagés; 4 . exploitations souterraines „5. localités ; 6. la progression des exploitations, 7 , future extension de l'exploitation.

Dans la partie droite de l'image on aperçoit la rivière Jiu avec des excavations qui se déroulent tout au long de celle-ci (le bassin de Rovinari) ; au centre, ce sont les excavations du bassin du Jilt, qui occupent l'espace collinaire très fragmenté, situé entre les vallées du Jiu à l'est et la vallée du Motru à l'ouest; à gauche de l'image ce sont les exploitations du bassin de Motru qui se développent au long de la rivière Motru et dans l'espace collinaire situé à l'est. À l'extrémité ouest se trouvent les exploitations du bassin de Mehedinti. Le bassin minier d'Olténie se continue vers l'est avec les exploitations du bassin de Berbesti (qui ne se trouvent pas dans cette figure) situées dans le département de Vâlcea.

\section{La dynamique de l'exploitation minière et ses conséquences paysagères}

Si l'exploitation du lignite a permis le développement économique de cette région, elle a laissé aussi de profondes traces dans le paysage. Les transformations apportées au paysage, par l'exploitation du lignite à ciel ouvert, sont caractérisées par J. Gardner (2001) de la manière suivante : «l'exploitation minière s'accompagne d'un défrichage total du sol, d'une modification radicale du paysage et d'un dérèglement total de l'écosystème ». Ce type d'exploitations représente une véritable provocation pour les défenseurs de l'environnement par la multitude de types d'impact qu'elle engendre. L'impact le plus visible est, sans aucun doute, la modification de la topographie avec l'apparition des grandes excavations aux versants raides dominées par des terrils tabulaires «immenses surfaces sinistres présentant un paysage lunaire, labouré, sur lesquels le vent soulevait des nuages de poussière" (Deshaies, 2003). S'y ajoutent: la destruction des nappes phréatiques, la destruction du sol, la destruction de la végétation, le déplacement de la population, qui justifient que l'on considère, une mine à ciel ouvert comme " un paysage désolé, dénudé et dépourvu d'êtres vivants ». 


\section{A. La destruction des nappes phréatiques} De nouvelles formes de relief sont mises en place et leurs caractéristiques topographiques sont déterminantes dans le cadre des plans de réaménagement et de reconstruction des paysages. Les carrières sont en forme d'amphithéâtre avec une profondeur qui varie entre $50 \mathrm{~m}$ et $175 \mathrm{~m}$, délimitées par des versants très raides avec des inclinaisons qui parfois atteignent jusqu'à $50-70^{\circ}$. Les terrils ont en général une forme allongée occupant plus d'espace, et sont situés dans le lit majeur, sur des terrasses ou à flanc de coteaux. Les matériaux qui les composent sont représentés par des argiles, marnes et sables et sont déposés d'une manière chaotique, ce qui rend ces formes de relief instables. Si on ajoute à ces matériaux l'absence de végétation et le régime pluviométrique, avec une fréquence élevée des précipitations orageuses, on comprend le pourquoi de la vulnérabilité de ces nouvelles formes de relief. Des 
affaissements se produisent tout au long des galeries souterraines, d'autant plus que les mines souterraines sont en grande partie fermées faute de rentabilité.

\section{Des problèmes sociaux}

39 Si les blessures du relief peuvent être dissimulées sous des forêts verdoyantes ou sous des plans d'eau étendus, les blessures sociales causées par les exploitations sont les conséquences les plus lourdes de cette activité. Représentées par la disparition des villages, par la destruction des églises ou des monuments architecturaux, des cadres familiers, mais surtout par la destruction des liens sociaux entre la population, les problèmes sociaux sont difficiles à résoudre. La destruction des villages accompagne l'activité minière depuis le début et continue aussi à présent. La plus affectée est la population du bassin minier de Rovinari où, jusqu'aux années 2000, 2090 maisons ont été détruites, nécessitant le déplacement de 5270 personnes (Calugaru, 2004). Il est vrai qu'on peut difficilement comparer ce nombre avec les 80000 personnes (Deshaies, 2003) déplacées en RDA (République Démocrate d'Allemagne) pour la mise en place des exploitations de lignite à ciel ouvert. Mais si l'on précise que cela représente $53 \%$ de la population habitant dans ce bassin, on peut se rendre compte de l'ampleur de ce phénomène au niveau local. Même si les villages détruits sont reconstruits sur des sites nouveaux, la rupture psychologique créée par la dissolution des anciennes communautés ne peut pas être comblée.

\section{La reconstruction du paysage minier}

L'image "noire et sale " caractéristique des régions minières et leurs conséquences environnementales, économiques ou sociales, ne s'arrête pas à la fermeture des mines. Des problèmes surgissent alors, problèmes dont on ne se rendait pas compte pendant le déroulement de l'exploitation et des nouvelles structures doivent être mises en place pour gérer ce qu'on appelle «le risque de l'après mine». La reconstruction des paysages représente une tache difficile pour n'importe quel pays, société ou système économique.

41 En Roumanie le cadre législatif de l'activité minière est représenté par la loi des mines no. $85 / 2003$, qui remplace la loi $61 / 1998$, et l'autorité habilitée pour son application est l'Agence Nationale des Ressources Minérales. Conformément à cette loi, toute activité minière est interdite sur des surfaces où sont implantées des monuments historiques, culturels, religieux, des sites archéologiques, ou sur des surfaces de protection sanitaire pour les sources d'alimentation en eau. Le Programme principal, qui établit les politiques et les mesures de reconstruction, est la Stratégie Minière définie par l'Arrêté Gouvernemental $n^{\circ}$ 615/2004. La Stratégie Minière combine des mesures économiques, structurelles et sociales et ressemble beaucoup à la "Kohlengesetz», plan de reconversion de la Ruhr (Holz, 1992).

Conformément à ce cadre législatif, la réhabilitation des surfaces exploitées avant 2003, est une obligation du Ministère de l'Agriculture des Forêts, des Eaux et de l'Environnement, à travers les directions départementales de spécialité et avec des fonds provenant du budget de l'Etat. Pour les surfaces exploitées après 2003, la réhabilitation est une obligation de la Compagnie minière qu'elle doit réaliser avec ses 
propres fonds. Les Compagnes minières doivent réaliser des dépôts bancaires, une fois que l'activité d'extraction commence, comme garantie pour la réhabilitation.

En Roumanie, 60 \% de la surface des friches minières recensées se trouve dans la région minière d'Olténie. Leur intégration soulève de nombreux enjeux en matière d'aménagement du territoire et de développement: des enjeux environnementaux, à cause des nombreux polluants qu'elles peuvent abriter, des enjeux fonciers, liés à la dévalorisation des terrains, et des enjeux esthétiques, liés à la crise paysagère (Joly, 2001). À ces déséquilibres viennent s'ajouter ceux déterminés par la restructuration de l'industrie minière, après 1997, qui a engagé la région dans une situation de crise, la plus difficile à surmonter étant la crise sociale et économique.

Avant 1990, les actions de réhabilitation ont été réalisées en mode irrégulier, en fonction des ressources financières que l'entreprise minière recevait de la part de l'État. Dans le nouveau cadre politique d'après 1990, les travaux de remise en état et de reconstruction, sont définis bien avant l'ouverture des carrières à l'aide d'études d'impact, intégrées au dossier d'autorisation d'ouverture. Dans la région minière d'Olténie, les objectifs de la réhabilitation sont surtout la remise en état agricole et le reboisement. Là où les surfaces excavées ne peuvent pas être complètement rebouchées, à cause du volume de matériel extrait, des plans d'eau (fig. 5) ont été créés. Ces choix de la Compagnie Nationale du Lignite d'olténie correspondent d'une part, à la demande des collectivités locales, qui souhaitent récupérer les terrains dont elles ont été dépossédées et d'autre part aux caractéristiques générales de la région. Depuis le début de l'exploitation minière et jusqu'en 2000, 2704 ha ont été réintroduits dans le circuit productif, 1725 ha de terrains agricoles ont été créés, 980 ha ont été reboisés, et quelques 3500 ha sont en cours de réaménagement (Baican, Vulpe, Ianc, 2004).

Que ce soit des forêts, des surfaces lacustres, des surfaces agricoles ou autres, à l'achèvement du processus de reconstruction, dans les régions avec exploitations à ciel ouvert, le "paysage de succession minière » est totalement différent du paysage qui existait auparavant. Il se caractérise par de notables différences topographiques, des collines artificielles formées par l'accumulation des stériles à la place des vallées, des surfaces planes à la place des collines.

Photos 1 à 4 : Les mêmes blessures paysagères, des paysages de succession minière différents Quand on parle des caractéristiques paysagères des exploitations à ciel ouvert, elles sont les mêmes, que ce soit en Roumanie (mine de Husnicioara) ou en Allemagne (mine de Garzweiler). Quand on parle de processus de réhabilitation la situation change : le lac Rovinari se situe sur la rive gauche de la rivière Jiu ; c'est une ancienne excavation remplie par la remonté naturelle des nappes phréatique et non par un processus d'aménagement planifié comme dans le cas du lac de Cospuden, situé en bordure sud de Leipzig, en Allemagne. 


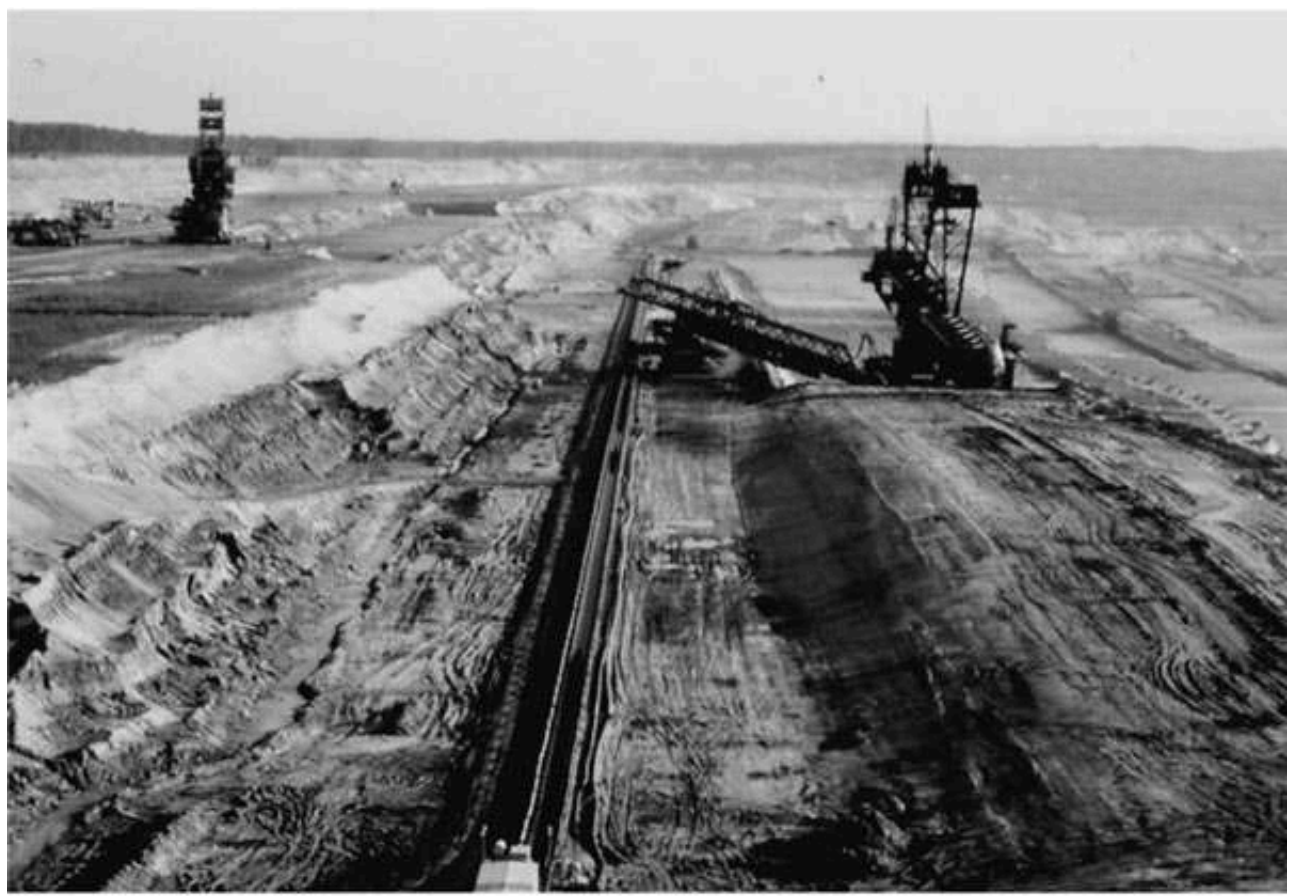

Exploitation de Garzweiler, près de Cologne

Photo : M. Deshaies (2003)

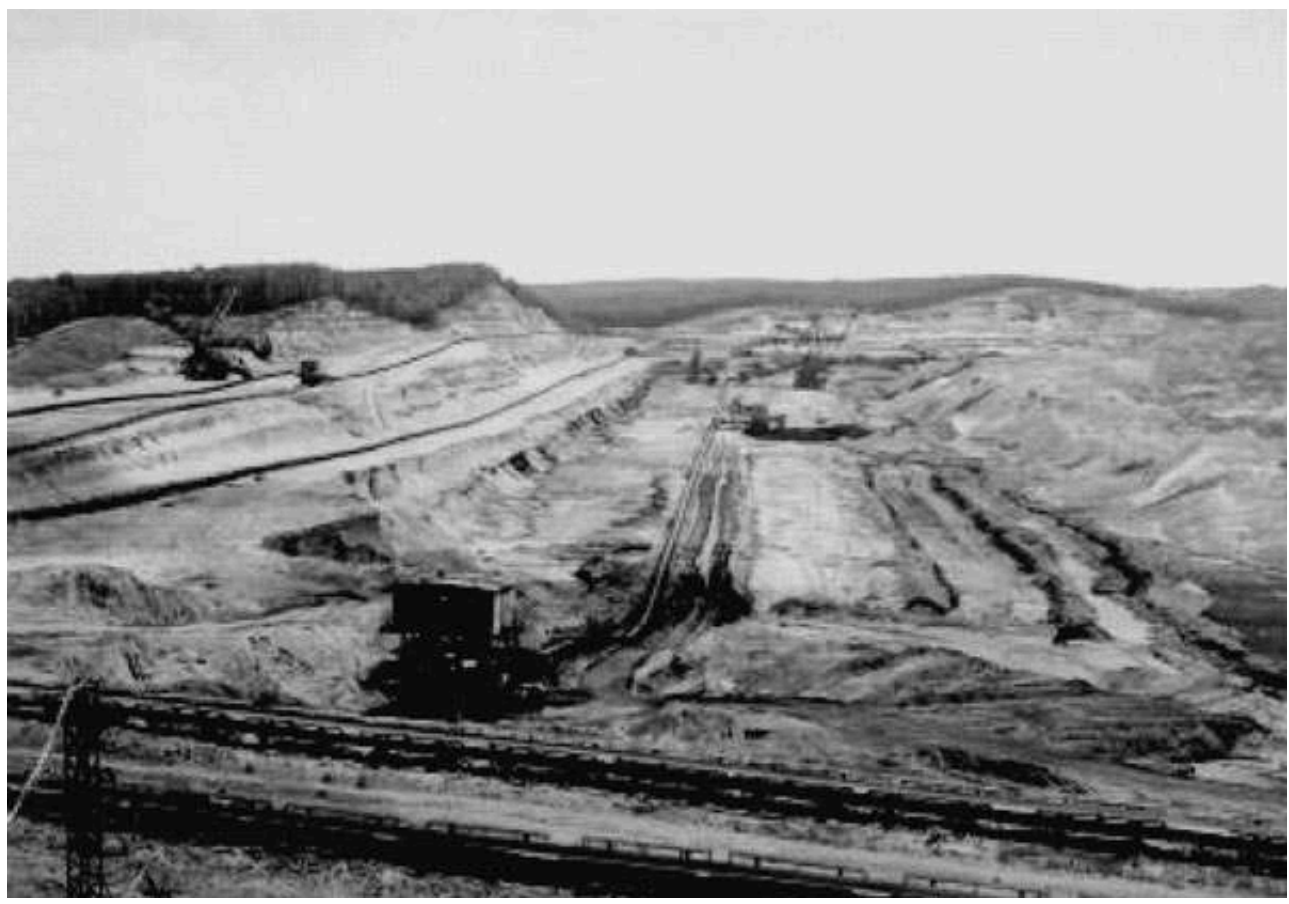

Mine de Husnicioara

Photo : CNLO (2003) 


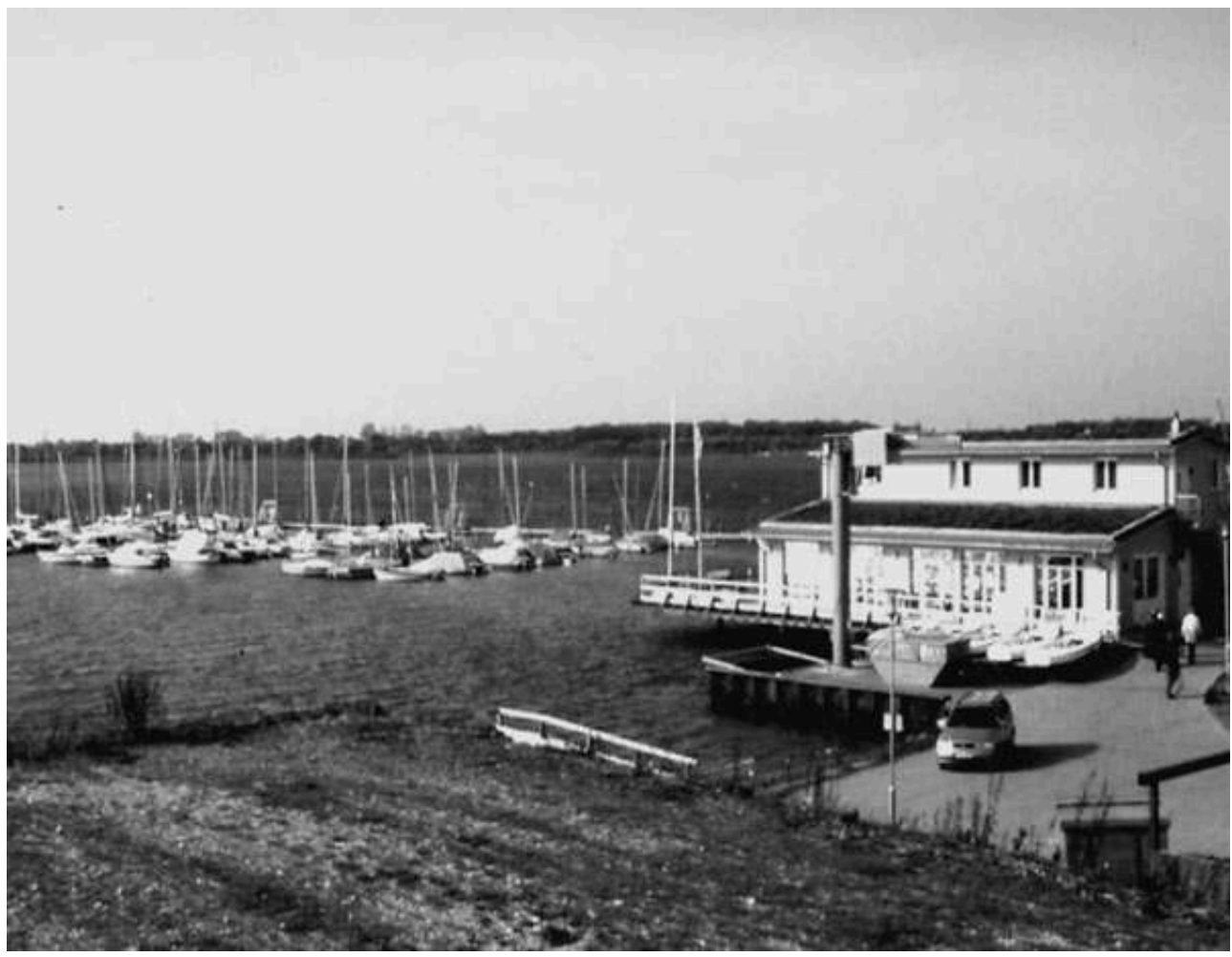

Lac de Cospuden

Photo : M. Deshaies (2003)

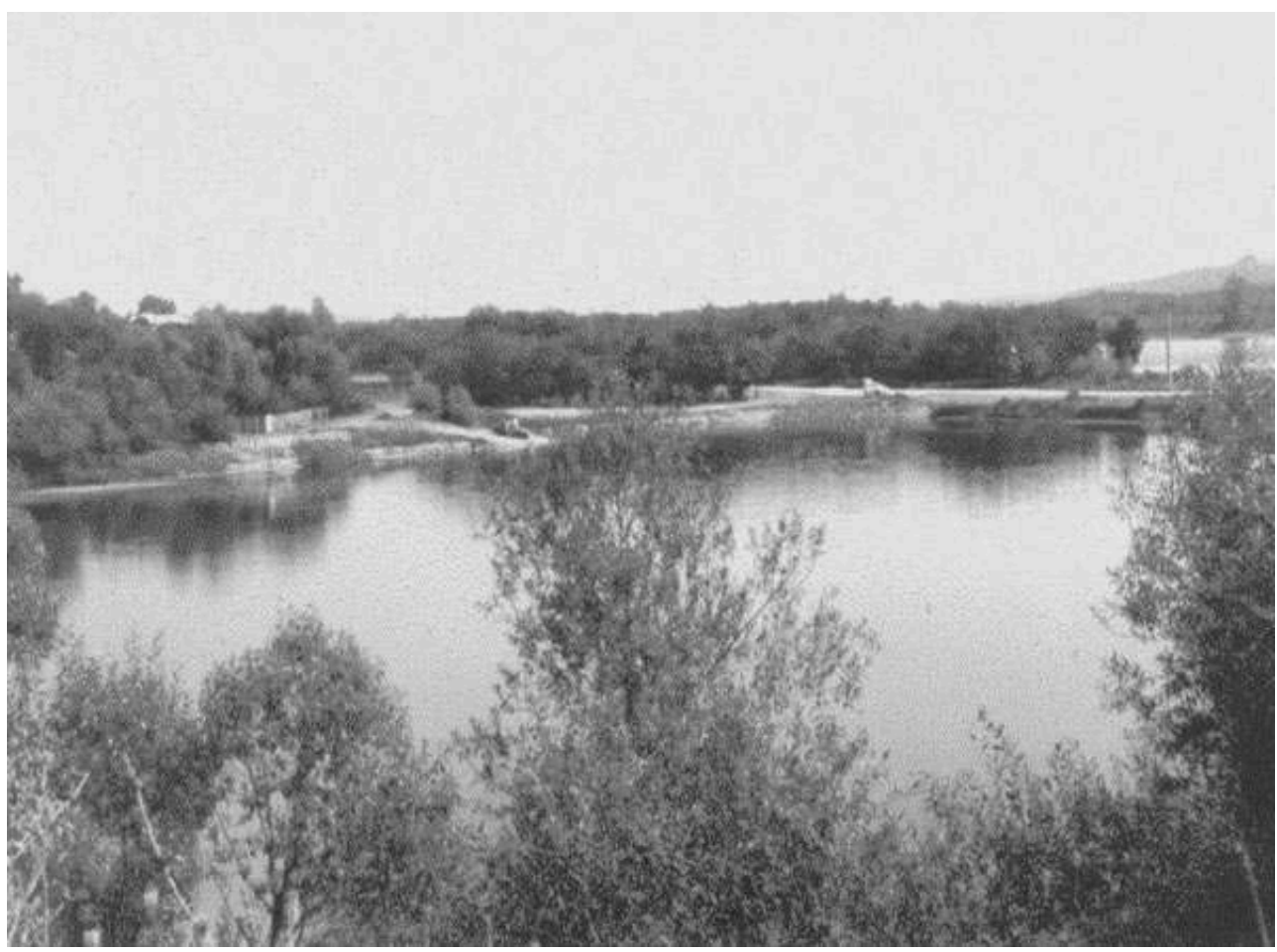

Lac Rovinari

Photo : C. Calugaru (2003)

Les traumatismes sociaux et paysagers causés par l'activité minière à ciel ouvert sont en général les mêmes (Photos 1 et 2 - les mines à ciel ouvert), quel que soit le système 
économique ou politique dans lequel on se trouve. Par contre, les méthodes de reconstruction et les paysages de succession minière sont strictement dépendants de ceux-ci (Photos 3 et 4 - les lacs). La mise en pratique des stratégies et des programmes de réhabilitation dépendent largement des ressources financières disponibles. La Roumanie, comme d'autres pays engagés sur le chemin de la réhabilitation, bénéficie des aides européennes (la Banque Européenne pour la Reconstruction et le Développement, BERD) et mondiales (la Banque Mondiale). L'Union Européenne a récemment attribué 5,2 millions d'euros aux comtés de la Vallée du Jiu et Gorj pour favoriser la réhabilitation de ces régions. Les fonds sont fournis dans le cadre du Programme MARR (réhabilitation des régions touchées par l'extraction minière).

\section{Conclusion}

Pendant la deuxième moitié du $\mathrm{XX}^{\mathrm{e}}$ siècle la région d'olténie s'est vue construire une nouvelle identité. Cette identité, elle la doit à la réalité géologique, la présence des gisements de charbon et à la réalité politique d'après la deuxième guerre mondiale qui a mis en exploitation ces gisements. Dans son développement territorial, l'industrie minière a englouti tout sur son passage, des terrains agricoles, des forêts, des collines, des vallées, des villages, a mis en place un nouveau type de paysage. À partir de 1956, l'évolution du paysage minier a suivi une progression nord-sud et est-ouest, au fil de la découverte et de la mise en valeur des gisements. On assiste d'un côté à un développement économique et à un accroissement démographique qui se sont soldés par l'apparition de villes nouvelles, et d'un autre côté à l'apparition des problèmes environnementaux: destruction du relief, abaissement des nappes phréatiques, déforestation, destruction $\mathrm{du}$ sol.

Même si dans la région d'Olténie l'activité minière reste encore une réalité, en comparaison avec d'autres régions du pays, elle est aussi confrontée, à partir de 1997, au processus de restructuration et de reconversion qui caractérise cette activité à travers l'Europe. La restructuration continue et dépend à la fois des forces extérieures qui agissent sur une région ou sur un pays et des forces endogènes de celle-ci. Si le rôle des systèmes extérieurs d'acteurs a été déterminant jusqu'à nos jours pour le développement de la région, étant à l'origine de la mise en exploitation des richesses «en sommeil » du bassin charbonnier (pour citer Holz J.-M. qui fait référence au bassin Ruhr), aujourd'hui ce sont les acteurs locaux qui doivent agir dans une logique de développement durable, "penser global, agir local ».

La reconstruction du paysage, dans les limites de la région étudiée, a démarré tôt, presque en même temps avec l'activité extractive, mais la priorité donnée à l'exploitation du lignite et l'absence des moyens financiers a fait que le pourcentage des surfaces reconstruites soit réduit. Aujourd'hui le nouveau cadre politique, économique et institutionnel, favorise la prise en compte des problèmes environnementaux mais l'héritage laissé par l'ancien régime en termes d'identité sociale, des caractéristiques économiques et de morphologie paysagère pèse lourd sur l'évolution ultérieure de la région d'olténie.

La volonté de reconstruire les espaces endommagés et les politiques qui existent au plan international pour la réhabilitation assure le passage du stade des projets au stade des perspectives, mais ce sont les ressources financières qui permettent de transformer les perspectives en réalité. Les politiques nationales et régionales de réhabilitation des 
paysages miniers restent souvent une traduction spatiale des politiques économiques et moins des politiques environnementales. Comment concilier développement économique et qualité de l'environnement? C'est là l'enjeu le plus important de la réhabilitation du paysage minier.

\section{BIBLIOGRAPHIE}

BAICAN G., VULPE I., IANC I. (2004). - « Reabilitarea si introducerea in circuitul productiv a zonelor ocupate de haldele de steril rezultate in urma exploatarii rezervelor de lignit si carbune brun din Romania », Buletin Informativ, No. 1-2/2004, MAPPM.

BAUDELLE G. (1994). - « Le bassin minier du Nord-Pas-de-Calais après le charbon : la difficile gestion de l'héritage spatial », Hommes et Terres du Nord, Revue de l'UFR Géographie Aménagement, Université des Sciences et Technologie de Lille, 1994/1.

CALUGARU C. (2004). - Les changements de l'environnement dans le bassin sous-carpatique et piémontais $d u J i u$, Thèse de doctorat, l'Institut de Géographie de l'Académie Roumaine, $420 \mathrm{p}$.

DESHAIES M. (2001). - « La réhabilitation des paysages dans l'ancienne région minière du rebord oriental du Harz », Revue Géographique de L'Est, Tome XLI, 1-2, p7-15.

DESHAIES M. (2003). - Mines et énergie en Allemagne : enjeux environnementaux et paysages, CERPA, Université de Nancy 2, http://fig-st-die.education.fr DE MARTONNE E. (1931). - Europe centrale, T. IV (2 ${ }^{\mathrm{e}}$ partie) de la Géographie Universelle, A. Colin, $845 \mathrm{p}$.

GARDNER J. (2001). - «Restaurer des sites miniers pour atteindre des objectifs d'utilisation des terres : L'extraction de la bauxite dans la forêt de jarrah en Australie Occidentale », Unasylva, 207, vol. 52

HOLZ J.-M. (1992). - Gérer l'espace. L'action des collectivités locales dans l'aménagement et la dynamique d'une région européenne : la Ruhr, Collection Etudes $\mathrm{N}^{\circ} 4$, Presses Universitaires de Perpignan.

JOLY N. (2001). - Les territoires renouvelés de l'après charbon. Loisir et cadre de vie dans le Nord-Pas-deCalais et la Ruhr, Thèse Université de Sciences et Technologies de Lille1, sous la direction de D. Paris, UFR de Géographie Et d'Aménagement.

LHOMEL E. (1984). - «Les problèmes de l'environnement en Roumanie », Le Courrier des Pays de l'Est, no. 281.

PALCU M. (2003). - « L'impact du drainage minier sur l'aquifère dacien inférieur en département Gor $\mathrm{j}$ ». In : 100 de ani de hidrogeologie moderna in Romania, Editura tehnica Bucuresti.

VON HIRSCHHAUSEN B. (1997). - Les nouvelles campagnes roumaines. Paradoxes d'un retour paysan, Belin, Paris.

World Energy Council East-West European Energy Programme (2000). - Restructuring and privatizing the coal industries in central and eastern Europe and the CIS, Published by Regency House, London. 


\section{NOTES}

1. La carte du Specht qui date de 1790. Cette carte fait partie de premières représentations cartographiques de la région d'olténie réalisées par les autrichiens après l'incorporation de cette région à l'empire austro-hongrois en 1718.

\section{RÉSUMÉS}

Comme dans tous les pays d'Europe centrale, l'exploitation du lignite en Roumanie a décliné, mais elle continue à conserver une place importante pour la production d'électricité. L'activité extractive, particulièrement les exploitations à ciel ouvert, est considérée comme une des activités qui marquent le plus les paysages. Des villages, des forêts, des rivières disparaissent et à leur place de nouveaux paysages voient le jour. L'exploitation du lignite a ainsi créé un type de paysage avec des caractéristiques particulières. À la fin de l'exploitation, des projets de réhabilitation sont mis en place et le paysage se transforme de nouveau. Des politiques, des programmes et des budgets sont consacrés à la reconstruction et à la reconversion des anciennes régions minières. L'article examine la situation actuelle des exploitations minières en Roumanie, un pays qui après 45 ans de communisme s'est engagé dans un processus de reconstruction et de reconversion à tous les niveaux, l'industrie minière en faisant partie.

As in all lands in Central Europe the extraction of lignite in Romania has decreased, but it still remains a great place for electric production. The extraction in open-cast mines is regarded as an activity which has the greater impact on landscapes. Villages, forests, rivers fell victim of the excavators and on their places new landscapes are shaped. The extraction of lignte has shaped a category of landscape with its own characteristics. At the end of the extraction projects of remediation are developed and the landscape undergoes transformation. Concepts, programms and financial means are employed for the restoring and the restructuring of mining regions. The article presents the situation of mines in Romania, a land which after 45 years communism has entered in a restructuring process on all scales, mining industry is a part of this process.

Wie in allen mitteleuropäischen Ländern ist der Braunkohlenbergbau niedergegangen, aber er hat noch heute einen großen Platz für die Stromerzeugung. Der Bergbau, insbesondere die Tagebaue ist eine der Aktivitäten, die den größten Eingriff in den Landschaften verursacht. Dörfer, Wälder, Flüsse werden abgerissen und auf ihrem Platz entstehen neue Landschaften. Durch den Braunkohlenbergbau ist eine Landschaft mit besonderem Kennzeichen entstanden. Am Ende des Bergbaus werden Rekultivierungsprojekte durchgeführt und die Landschaft wandelt sich wieder. Politik, Programme und finanzielle Mittel stehen zur Verfügung für die Umstrukturierung der ehemaligen Bergbaugebiete. Der Artikel stellt die heutige Lage der Bergwerke in Rumänien dar, ein Land, das nach 45 Jahren Kommunismus sich in einem Wiederaufbau- und Umstrukturierungsprozess in allen Gebieten befindet, der Bergbau bildet eine Teil dieses Prozesses.

Landschaft, Bergwerk, Tagebaue, Halden, Braunkohle, Rekultivierung, Wiederaufbau, Rumänien 
INDEX

Mots-clés : paysage, mine, exploitations à ciel ouvert, terrils, lignite, réhabilitation, reconstruction, Roumanie

Keywords : landscape, mie, open-cast mines, tip-heaps, lignite, remediation, restoring, Romania

\section{AUTEUR}

CORINA CALUGARU

Post Doctorante - ENS-LSH Lyon - 15 Parvis René Descartes 69342 Lyon -

coricalugaru@yahoo.com 